\title{
Cultura institucional PaRa la transición: COMANDO AÉrEO de COMBATE $\mathrm{N}^{\circ} 2$
}

\author{
Teniente Coronel (RA) ALEXANDRA GUZMÁN DURÁN
}

\section{INTRODUCCIÓN}

El presente documento sintetiza uno de los componentes investigativos desarrollados con el objeto de comprender los elementos culturales y de construcción de sentido empleados por los Oficiales y Suboficiales adscritos al Comando Aéreo de Combate $\mathrm{N}^{\circ} 2$-CACOM 2- para pensar prospectivamente el futuro cercano de la Fuerza Aérea Colombiana y de esta unidad militar en particular. Dicho ejercicio se enmarca en un proyecto de investigación más amplio $^{2}$, que busca explorar los elementos psicosociales que determinan y configuran al personal que hace parte de Apiay.

El ejercicio tiene un marco histórico que legitima y justifica su realización y que define el objeto principal de este artículo de investigación: El proceso de paz que se desarrolla con la organización armada de las FARC presupone unas transformaciones institucionales muy importantes del Estado colombiano. Entre las instituciones que han sido llamadas a transformar su estructura, están las Fuerzas Militares, quienes desde ya han iniciado a estudiar este proceso, y a prepararse para estas transiciones.

Sin embargo, los procesos de pedagogía al interior de las Fuerzas no han logrado avanzar con la misma velocidad con la que lo hace el proceso de negociación y la información -falsa o verdadera- que se ha divulgado a partir de los eventuales acuerdos que se logren. Esta falencia ha ocasionado malestares, desinformaciones y rumores, en los que se cuestiona la seguridad jurídica y económica de los miembros de la Institución; y profundas críticas a lo que se viene negociando.

Psicóloga de la Reserva Activa de la Fuerza Aérea Colombiana e investigadora del Grupo Análisis y Contexto.

2 Esta investigación se complementa con un análisis de la Empatía, emociones y estrategias de regulación emocional en diferentes poblaciones afectadas por el conflicto armado en Colombia, la cual fue realizada en conjunto con los psicólogos PhD Hernán Camilo Hurtado y con el PhD Carlos Gantiva; cuyos resultados serán publicados en un informe de investigación más amplio. 
Para poder subsanar este vacío es necesario reforzar los procesos de pedagogía para la paz al interior de las Fuerzas. Para ello, hay que dimensionar que cuando se habla de pedagogía no se limita al acto de transmitir información, sino que se entiende en su sentido más amplio: comprensión de las formas de pensar de un grupo, y a partir de ello, generación de estrategias comunicativas y didácticas acordes para el público específico (RoJANO, 2008; ORTEGA, 2012).

Esto quiere decir, que antes de generar acciones pedagógicas y de sensibilización, es necesario comprender cómo entienden los miembros de la Fuerza Aérea Colombiana este proceso de paz; es decir, cuáles son los referentes políticos y sociales desde los que piensan esta negociación. Lo que en últimas, se entiende como la comprensión de la cultura para la paz de los militares adscritos a la Institución.

Comprender esta cultura es de gran utilidad para liderar de forma efectiva los procesos de transición de las Fuerzas Militares; pero también para romper el imaginario colectivo que señala que estas instituciones castrenses son opuestas y contrarias a la búsqueda negociada de la paz.

\section{METODOLOGÍA}

Para el desarrollo de este proyecto de investigación se realizó un ejercicio investigativo fundamentado en las premisas epistemológicas de la etnografía, entendiendo que el objeto central de esta rama del conocimiento son las formas de actuar y de pensar, determinados por un referente cultural concreto.

Así, la investigación etnográfica trata de develar los elementos culturales (tradiciones, rituales, ideologías-idiosincrasias, y pautas de comportamiento estandarizadas) que justifican y explican la forma en la que piensa y actúa un determinado colectivo, y que les lleva a proyectar estereotipos, prenociones y formas de comprender la realidad social (AGUiRRE, 1995).

En el caso concreto de esta investigación consistió en develar las formas de pensamiento históricamente utilizadas y referidas por los miembros de la Fuerza Aérea Colombiana, para poder comprender por qué se reacciona de una manera determinada frente a los procesos de negociación que se están desarrollando. Así mismo, una determinación de la estrategia pedagógica que se debe desarrollar para generar impactos efectivos en la cultura institucional de los miembros de la Fuerza Aérea Colombiana.

Todo esto se derivó en un procedimiento de investigación específico, que permitió comprender los elementos que definen esta cultura institucional. Este procedimiento se fundamenta en los postulados de los enfoques cualitativos (Delgado, 2006), y se estructuraron en una estrategia puntual desarrollada a partir de unos grupos focales realizados con un personal militar heterogéneo 
(en términos de grados, tiempo de permanencia en la Institución y en la Unidad militar, entre otros factores); así como una revisión documental.

\section{HALLAZGOS}

Fruto del ejercicio investigativo emergieron unos temas concretos sobre los que había coincidencias y acuerdos entre los distintos grupos consultados. Estos hallazgos cabe entenderlos como narrativas comunes que empiezan a determinar rasgos comunes e identitarios del personal adscrito a CACOM 2. Por supuesto, alrededor de estos temas transitan unos puntos de disenso y debate, pero que cuantitativamente no son significativos ni incidentes para impedir que se configuren estos acuerdos.

Con el ánimo de ordenar estas narrativas, acorde con el procedimiento metodológico empleado, se presentan en tres grandes grupos temáticos; estos temas se articulan plenamente con los debates propios del ámbito de la transición, y su diagnóstico es una pieza fundamental para futuros ejercicios pedagógicos que se quieran desarrollar con este personal militar. Se hace una exposición detallada de cada uno de estos debates.

\subsection{LO POLÍTICO EN LO MILITAR: UNA MIRADA A LA NEGOCIACIÓN DE PAZ}

El primer gran tema sobre el que emergió un acuerdo tácito entre los participantes, es la posición favorable a que se alcance la paz en el país, entendiendo que ésta no será resultado exclusivo de intervenciones militares, y que implicará acuerdos políticos y jurídicos con las partes. Sin embargo, este apoyo viene acompañado de profundas desconfianzas sobre lo que se está pactando en los Diálogos de la Habana.

Esta desconfianza es el resultado de una incredulidad sobre los canales de difusión y comunicación que se han desplegado para socializar los acuerdos a los que se han llegado; responsabilizando de esta mala estrategia comunicativa al Gobierno Nacional y los medios de comunicación que, según varios de los consultados, tienen una posición amañada e interesada sobre el resultado final de los acuerdos.

Por supuesto, esta posición no es ligera y desinformada, sino que surge del conocimiento del terreno y de la realidad que ellos tienen, y que no ha sido recogida suficientemente en los informes mediáticos y académicos, fenómeno que ahonda y profundiza las desconfianzas y los recelos.

Es fundamental aclarar que el universo poblacional es absolutamente respetuoso de las decisiones tomadas por el Gobierno Nacional, y que acatan el 
resultado de los procesos, pero en el marco de un ejercicio netamente académico, se permiten manifestar estas dudas e inquietudes.

El primer grupo de dudas se teje entorno a la voluntad real de paz de la organización armada, basados en la labor de inteligencia realizada por tantas décadas, y que no permite apreciar con claridad esta voluntad. De hecho, es importante señalar que aquellos que tienen una relación directa con la especialidad de Inteligencia, fueron los que manifestaron mayores incredulidades y temores con el resultado final de esta negociación.

De este factor, y de la voluntad real que exprese este grupo, dependerán los posibles actos de perdón y de reconciliación que se presenten en el futuro; ya que no puede haber un acto sincero de perdón, si está fundamentado en desconfianzas y resquemores.

En segundo lugar, hay un temor generalizado por la suerte de los territorios que actualmente ocupa este grupo armado, ya que las posibilidades de que otros grupos armados ilegales los ocupen son bastantes altos, tal y como lo ilustra la historia reciente de la desmovilización de las Autodefensas Unidas de Colombia -AUC. Este temor no es particular de este grupo social, sino que se puede rastrear fácilmente en distintos informes académicos realizados en Colombia para determinar los riesgos de seguridad del posconflicto (Observatorio del Programa Presidencial de Derechos Humanos y DIH, 2012).

Esto se concadena con un tercer temor, en el que se preguntan por el futuro inmediato de las bases de las FARC, esos desmovilizados que no van a tener espacios de representación ni inserciones mediáticas en la sociedad civil, y que no conocen otra vida que las armas y la violencia. Allí, consideran que si no se hace un ejercicio serio e integral, se corre el riesgo de que estas personas reincidan en la violencia y la criminalidad a nombre de otros grupos ilegales, con graves consecuencias socio jurídicas derivadas del ámbito del Derecho Internacional Humanitario que no cobijaría a estas organizaciones y que dejaría en un vacío jurídico a la Fuerza Aérea Colombiana (ReTtBerg, 2012).

Este punto es muy importante en la concepción etnometodológica (ESQUIVEL OCADÍZ, 2016) que hacen ellos sobre las FARC, porque entienden que no pueden equipararse las bases con la cúpula de la organización, siendo esta última la que gozará de todos los derechos y reconocimientos propios de las negociaciones. Por ello, entienden que estas bases han sido instrumentos históricos de esas cúpulas, que los han llevado a cometer actos atroces; ante ello, la aplicación de la justicia transicional puede ser más benevolente con esas bases, pero no tanto así con los máximos responsables de los hechos más violentos, según consta en los ejercicios investigativos realizados.

Finalmente, emergen unas desconfianzas materiales y concretas sobre el futuro inmediato de la institución: emergiendo categorías tales como las afectaciones sobre elementos pensionales de los miembros de la Fuerza Pública, reducción de 
ingresos por cuenta de la eventual desaparición de la "prima de orden público"; y en las posibles acciones judiciales descontextualizadas y tendenciosas que podrían realizarse contra los militares y policías. Este último punto es fundamental, porque significa un temor por la seguridad jurídica de los miembros de las Fuerzas Militares, pero también una afrenta contra el honor militar y contra la dignidad de las instituciones castrenses.

\subsection{ELEMENTOS DE AUTODEFINICIÓN: REPRESENTACIÓN DE LO MILITAR}

Una de las conclusiones generales a las que llegaron los entrevistados, es que hay un profundo desconocimiento por parte de la sociedad civil sobre las tradiciones, costumbres y roles militares: lo cual incide en que en ocasiones se juzgue de forma errada el proceder de la Institución. Sobre esta situación se presentó un debate muy interesante del que surgieron dos posiciones:

1. La sociedad desconoce a las Fuerzas Militares porque los órganos (gobierno, medios de comunicación, sector académico, entre otros) que se han encargado de presentarlas, lo han hecho de forma incorrecta y, en ocasiones, tendenciosa e ideológicamente sesgada.

2. Las mismas Fuerzas Militares han fallado a la hora de dar a conocerse a la población, encerrándose mucho en sus instalaciones militares, y sin construir una estrategia comunicacional que impacte a los distintos públicos. Así mismo, ha adolecido de escritores internos o afines, que se ocupen de contar su historia a las generaciones futuras.

Como sea, este desconocimiento incide en que la sociedad juzgue o malinterprete acciones propias del mundo militar, y que tipifique estos comportamientos como arbitrarios o arcaicos. Por ejemplo, un fenómeno tan importante -para la población con la que hizo la investigación-como lo es el honor militar, siempre resulta difícil de explicar a una persona externa a estas instituciones.

Este "honor" está presente en todo el proceder y doctrina de la Fuerza Aérea de Colombia, y sus integrantes lo encuentran en la vocación para tomar esta opción de vida, en la forma en la que se procede en el día a día, en la "mística" con la que se emprenden las tareas y responsabilidades, y en la disposición de servicio y de sumisión institucional. Por ello, fue muy recurrente que en la lectura propia que realizaron estos señores Oficiales y Suboficiales, el término honor emergiera siempre que debían definirse (GONZÁLEZ, 2007).

De hecho, en este punto emergió un tema de debate: las diferenciaciones y rupturas generaciones ${ }^{3}$ que se pueden presentar dentro de la Fuerza Aérea

Este tema determinó uno de los criterios metodológicos de selección de la población para los grupos focales, ya que siempre se esperó contar con personal con gran trayectoria dentro de la Institución, pero también con la posición de aquellos que hasta ahora están entrando a la misma. 
Colombiana; tema importantísimo en este estudio porque habla de las posibles irrupciones y rupturas culturales que se pueden presentar dentro de la Institución.

Lo primero que debe señalarse, es que sí se encontró una autopercepción de la existencia de estas rupturas generacionales: manifestadas en las características de las personas que ingresan, en las aspiraciones de los miembros, y en el perfil de los mismos; a continuación, se analiza brevemente estos elementos.

Lo primero que señalaron los entrevistados se relaciona con las transformaciones sociohistóricas que ha vivido el país en los últimos años, y la imagen que proyectó la Fuerza Aérea Colombiana en cada uno de esos momentos. Así, es muy diferente que una persona ingresara a la Institución en la década de los años 80 , cuando el componente tecnológico era menor, y cuando el impacto mediático de las operaciones aéreas era reducido; o que una persona tomara la elección de enlistarse en la década de los años noventa, cuando las acciones que desarrollaban los grupos armados ilegales ponían en riesgo la vida de todos los ciudadanos y le daban una connotación de mayor peligro a este servicio; y por supuesto, es muy distinto el contexto actual, en el que la Fuerza ha generado operaciones aéreas muy exitosas y contundentes (Fuerza Aérea Colombiana, 2016) y cuando el ambiente social y político apunta a la eventual terminación del conflicto armado, por lo menos en la forma en la que hasta ahora se ha conocido.

Todos estos elementos contextuales, tienden a determinar un perfil general de la persona que ingresa a la Institución, señalándose que el componente militar ha venido disminuyendo, emergiendo una serie de jóvenes, mucho más profesionales y con aspiraciones de avanzar en su formación. Esto se define como una nueva generación de jóvenes que ponen el énfasis en los elementos profesionales, técnicos y burocráticos propios de su actividad, en desmedro de las actividades físicas, disciplinares y doctrinales propios de lo militar; lo primero no elimina lo segundo, pero sí le resta valía e importancia.

Finalmente, es importante señalar que no hay una valoración negativa o positiva de estos cambios, ya que señalan que no es un proceso exclusivo o particular de la Fuerza Aérea Colombiana, sino de la sociedad en general, y de las dinámicas de diferenciación propias de cada generación: los que hoy tienen más de 20 años de servicio dentro de la Institución, igual fueron jóvenes irruptores y transgresores de las costumbres cuando ingresaron a la Institución.

\subsection{Transformación de la Fuerza Aérea Colombiana: el PROCESO DE TRANSICIÓN}

La palabra con la que se afronta este proceso histórico es incertidumbre. Incertidumbre ante el futuro doctrinal, misional y del personal que hace parte de la Institución. Esta sensación no ha desaparecido aun cuando han recibido distintos procesos de sensibilización y capacitación sobre el rol de la Fuerza 
Aérea Colombiana en el eventual escenario del posconflicto, en parte porque consideran que los argumentos no son suficientemente claros, y en parte porque encuentran algunas incongruencias entre discurso y realidad.

Así mismo, como se expresó en un apartado anterior, subsisten unas desconfianzas y miedos sobre el futuro de la seguridad del país, después de que se cierren las negociaciones con las FARC, que llevan a que se problematice el alcance mismo de la transición, o la validez institucional de la misma. Así mismo, el confuso panorama que se cierne sobre el ELN, y su posible fortalecimiento tras la negociación con las FARC, refuerzan el recelo y desconfianza que se presenta en este punto.

Relacionado con esto, hay una tendencia a pensar que la posibilidad real del fin del conflicto o de la violencia va a ser lejana, en cuanto fenómenos como las "bacrim", el narcotráfico o los denominados delitos transnacionales van a ser elementos recurrentes en un futuro cercano y a medio plazo. Esto, acompañado de una incertidumbre jurídica y política sobre cuál será el rol que se le asigne a la Institución en un futuro cercano frente a estos flagelos sociales y de seguridad.

Sin embargo, son respetuosos del poder central y de las decisiones que allí se tomen, y lejos de buscar ser obstáculos o tropiezos en esta nueva fase de la historia del país, generan reflexiones sobre cuál podría ser el rol de la Fuerza Aérea Colombiana en ese nuevo contexto histórico, lo cual se puede apreciar en las siguientes propuestas.

En primer lugar, hay un llamado a recuperar los propósitos iniciales: proteger la soberanía nacional y monitorear las fronteras del país; para ello es necesario replantear las estrategias, modernizar algunos equipos y concentrar esfuerzos en esta importante tarea, máxime cuando hay escenarios complejos con países como Nicaragua y Venezuela.

En segundo lugar, la Institución tiene una fortaleza que ninguna otra fuerza aérea tiene: una amplísima experiencia en operaciones aéreas en el marco de un conflicto armado interno, lo que le permite transmitir este conocimiento a otros países y convertirse en un referente a nivel internacional; en particular, reconocen que CACOM 2 tiene grandes enseñanzas que compartir en este terreno.

Finalmente, aseguran que la Fuerza Aérea Colombiana ha sido central en los procesos de desarrollo de vastas regiones del país, en especial de aquellas que están más alejadas del centro y en donde es muy difícil el ingreso terrestre. A estas regiones, a las que a veces ni siquiera las instituciones del Estado pueden llegar, la Fuerza Aérea Colombiana ha generado presencia y asistencia en temas sociales (educación, brigadas de salud, y demás actividades concentradas en lo que se denomina la Acción Integral), asistencia humanitaria e interconexión de estas regiones con el resto del país. 
Basta señalar, por ejemplo, el caso de Satena y su compromiso con las regiones más apartadas y a las que ninguna aerolínea comercial llega; gracias a ésta se ha podido generar procesos económicos y de desarrollo, ligados con la aviación comercial y de transporte de pasajeros.

De esta manera, aunque hay unos miedos y resquemores ante el proceso que se está dando, tienen una mirada propositiva y abierta sobre la incidencia que podría tener la Fuerza Aérea Colombiana en estos nuevos contextos, sin perder de vista que ante una ruptura de los acuerdos o del cese al fuego bilateral, están plenamente preparados para retomar las operaciones aéreas que conduzcan a garantizar la seguridad de las distintas regiones del país.

\subsection{CACOM 2: APIAY Y SUS APORTES AL PROCESO DE TRANSICIÓN}

Hubo un consenso entre todos, a la hora de señalar la importancia estratégica que tiene CACOM 2 por su ubicación geográfica en el centro del país, lo que le permite alcanzar todas las posiciones del territorio nacional; no en balde, varias de las operaciones áreas más importantes han salido de esta Unidad, o han recibido apoyo estratégico de la misma, tal como lo evidencian las publicaciones académicas que se han desarrollado sobre este tema (MARín, et al, 2016).

A la vez, coinciden en señalar que esta Unidad tiene una "mística" especial, que hace que los más experimentados la definan como uno de los mejores Comandos Aéreos en los que han trabajado, porque es posible ver directamente el impacto regional y nacional que su labor ha generado. El tipo de aeronaves, el dinamismo de la operatividad y la permanente capacitación y actualización del personal hacen que Apiay sea altamente incidente en la seguridad del país (ECHANDÍA, 2013).

Por ejemplo, los que más años llevan en CACOM 2 (se entrevistaron a Técnicos Jefes que tienen más de 20 años en esta Unidad), recuerdan cómo era la situación de seguridad en la antigua vía Bogotá-Villavicencio, una vía donde era imposible transitar a ciertas horas del día, por la presencia de las FARC en municipios intermedios; situación que ya ha sido superada, y que ha permitido que el tránsito entre Bogotá y Villavicencio sea cada vez mayor, con todas las posibilidades de desarrollo, y posibilidades económicas para una importante zona de Colombia (CALDERón, 2015). Este cambio sólo fue posible por la mayor presencia de la Fuerza Pública en la región, y por supuesto, por la labor operativa y de inteligencia que prestó la institución, a través de CACOM 2.

$Y$ es que hay que entender que Apiay está ubicada en la puerta de entrada a la región de la Orinoquia, una zona muy rica en recursos naturales, pero con unas tasas de desarrollo muy bajas, y con una infraestructura vial y de servicios inadecuada e insuficiente (Fundación Ideas para la Paz, 2013). 
Por ello, la institución tiene una oportunidad privilegiada de ser incidente en la transformación económica de esta región del país, apoyando en la consolidación de una economía sólida y de un desarrollo social sostenible. La Fuerza Aérea Colombiana, por medio de CACOM 2 fue clave en la consolidación de la seguridad de la región en el peor momento del conflicto en estos territorios, ahora podrá ser incidente en su transformación económica en un eventual escenario de posconflicto (Observatorio del Programa Presidencial de Derechos Humanos y DIH, 2012).

Así mismo, Apiay está en una posición estratégica fundamental para monitorear las fronteras orientales, occidentales y al sur del país, lo que le permite ser trascendente en la protección de la soberanía del país, ante cualquier intento de transgresión que se pudiese presentar.

\section{REFLEXIONES FINALES}

El enfoque explicativo de este documento, giró en torno a tres niveles de análisis, acorde con los elementos macro y micro que emergen a nivel macro del análisis de los grupos focales, entre ellos están la transformación en la Fuerza, la pregunta por la identidad de lo militar y las relaciones cívico-militares. Esto se puede apreciar en la siguiente imagen, resultado de la entrevista a expertos en esta materia

\section{Imagen. Elementos macro emergentes}

\begin{tabular}{|c|c|}
\hline Transformación de la Fuerza \\
\hline Identidad de lo militar \\
\hline
\end{tabular}

Fuente: Elaboración propia a partir de entrevistas exploratorias realizadas (2016)

Lo interesante de este modelo de análisis, es que partiendo de las premisas etnometodológicas de Aguirre (1995) se puede establecer que hay un orden lógico para entender e interpretar la información encontrada, ya que para poder deducir cómo conciben la transición o el proceso de paz los miembros de la Fuerza Aérea Colombiana, primero hay que entender quiénes son y cuáles son sus rasgos característicos y que definen su identidad como militares.

En ese mismo sentido, cualquier apuesta pedagógica que se quiera desarrollar con esta población, tiene que partir de un conocimiento profundo y concienzudo de la población militar de la institución, para poder diseñar contenidos, estrategias didácticas y lenguajes que sean acordes y adecuados para este grupo poblacional 
y que permita que la sensibilización y construcción de contenido sea la adecuada para las necesidades informativas que ellos tienen.

Por ejemplo, como lo deja claro la lectura de GonzÁLEz (2007) y de las entrevistas realizadas, todo este ejercicio de conocimiento y pedagogía tiene que dimensionar lo que implica para esta población el "honor militar", ya que allí se establecen unos posibles puntos de encuentro, pero también unos elementos intocables y que generan altas sensibilidades en este grupo humano:

\begin{abstract}
"Lo humano está dotado de sentimientos que predisponen a la acción (1) y aquellos cuya conducta profesional más específica es el empleo de la fuerza con la finalidad de imponerse en el enfrentamiento y para ello, si llega el caso, eliminar a otros, deberían estar amparados en sólidas razones y firmes convicciones que legitimaran dicha conducta. El soldado no es un elemento material más de los que intervienen en una guerra, es un individuo dotado de razón y emoción, y que como todo ser humano intenta encontrar unos sólidos fundamentos morales y éticos a su profesión" (GoNZÁLEZ, 2007, p. 7).
\end{abstract}

Es desde este honor desde donde los miembros de la Fuerza Aérea entienden y comprenden el proceso de paz y las posibilidades de transición hacía un escenario de posconflicto; cualquier intento de construir discursos pedagógicos desde ejes conceptuales ajenos a este referente, corren el riesgo de no tener acogida dentro de la Institución, o peor aún, de generar desconfianzas y malestares dentro de estos Oficiales y Suboficiales.

Un segundo eje de conclusión derivado de este ejercicio investigativo, se estructura en torno a la concepción dual que tienen las personas entrevistadas sobre la Institución, señalando que ésta es apta y necesaria en tiempos de conflicto armado interno, pero que también lo es en tiempos de paz.

De hecho, son conscientes que el propósito institucional original no estaba enfocado a este tipo de conflictos, sino que tenían una misionalidad de protección de fronteras, y con ello, de la soberanía del país. Así mismo, en el quehacer de la misma, han emergido una serie de posibilidades de intervención en procesos de desarrollo con las comunidades, que ha dejado una impronta indeleble en estos hombres y mujeres.

Sin importar el contexto histórico, la Fuerza Aérea Colombiana fue, es y será fundamental para generar desarrollo y progreso en las zonas más alejadas e inhóspitas del país, allá donde otras instituciones del Estado no han podido llegar; allí donde el proceso de consolidación no ha iniciado.

En ese sentido, se entiende y explica una de las particularidades centrales de CACOM 2, ya que esta Unidad fue, es y será fundamental para el desarrollo de operaciones aéreas exitosas y efectivas, en razón de su privilegiadísima ubicación estratégica en el centro del país, y en cercanía de todas las fronteras del sur del 
país, lo que le permite ser punta de lanza en cualquier despliegue de poder aéreo que la Institución deba desarrollar.

Pero también Apiay es la Unidad militar que tiene mayores posibilidades para impulsar el desarrollo social y económico en la Orinoquia colombiana, la región llamada a ser la principal despensa agrícola del país, la generadora de hidrocarburos, y una zona de desarrollo demográfico sustentable y sostenible. Esto es un aporte histórico que este Comando le hace a la región, y en función de esto se sustentan las positivas relaciones que ha establecido con los actores sociales, políticos y económicos de la región.

De todo esto son conocedores los hombres y mujeres que trabajan y operan desde el Comando Aéreo de Combate $\mathrm{N}^{\circ} 2$, lo cual les hace sentir orgullosos del papel protagónico e incidente de esta Unidad militar en la historia reciente del país, y en la consolidación de la seguridad y el desarrollo en la región. Por ello, sin tapujos, y reconociendo que los otros Comandos y Grupos Aéreos son muy importantes, no dudan en señalar que "CACOM es el mejor Comando de la Fuerza Aérea Colombiana"'s.

\section{REFERENCIAS}

Aguirre Baztán, Ángel. (1995). La etnografía. Metodología cualitativa en la investigación sociocultural. España: Editorial Marcombo.

CALDERón, Emilse. (2015). Estados Unidos y el combate contra el narcoterrorismo como lineamiento de política exterior central en el vínculo con América Latina: el caso del modelo de cooperación con Colombia. Buenos Aires, Argentina, CERIR.

Delgado, Juan Manuel. (2006). Manual de métodos cualitativos. España: Fondo de Cultura Económica.

ECHANDía, Camilo. (2013). Punto de partida del debilitamiento de las FARC. En Revista Zero, Bogotá, Universidad Externado de Colombia.

Esquivel TrianA, Ricardo. (2016). Fuerza Aérea Colombiana y operaciones decisivas 1998-2015. En La Fuerza Aérea Colombiana y el termino del conflicto armado. Victorias desde el aire. Colombia, Impress Colombia.

EsQuivel OCADIZ, Alejandro. (2016). La Etnometodología, una alternativa relegada de la educación. En: Revista Iberoamericana para la Investigación y el Desarrollo Educativo: RIDE, Vol. 6, No. 12.

$4 \quad$ Fragmento de entrevista realizada en los grupos focales desarrollados. 
Fuerza Aérea Colombiana. (2016). LaZona de distensión y su recuperación por parte de la Fuerza Aérea Colombiana. En La Fuerza Aérea Colombiana y el término del conflicto armado. Victorias desde el aire. Colombia, Impress Colombia.

Fundación Ideas para la Paz. (2013). Hoy y ayer del Bloque Oriental de las FARC. Colombia, Fundación Ideas para la Paz.

GonzÁLEZ, J. A. M. (2007). Aproximación al concepto de moral militar. Boletín de Información, (298), 7-19.

Marín Ramírez, Adriana; Castiblanco Durán, Camilo; Castrillón Riascos, Javier y Cotrina Acosta, Andrés. (2016). La Zona de distensión y su recuperación por parte de la Fuerza Aérea Colombiana. En La Fuerza Aérea Colombiana y el término del conflicto armado. Victorias desde el aire. Colombia, Impress Colombia.

Ortega Valencia, Piedad. (2012). Pedagogía y alteridad. Una Pedagogía del Nos-Otros. En: Revista Virtual Universidad Católica del Norte, núm. 35, febrero-mayo. Fundación Universitaria Católica del Norte Medellín, Colombia

Observatorio del Programa Presidencial de Derechos Humanos y DIH (2012) El Caguán, Colombia, Presidencia de la República.

Rettberg, Angelika. (2012). Caguán 10 años: Más allá de un proceso de paz. Bogotá, Documentos del Departamento de Ciencia Política N 16, Universidad de los Andes.

RoJAno Mercado, Jairo. (2008). Conceptos básicos de pedagogía. En: REDHECS: Revista electrónica de Humanidades, Educación y Comunicación Social, Año 3, No .4

SALAS, Luis Gabriel. (2014). Lógicas territoriales y relaciones de poder en el espacio de los actores armados: un aporte desde la geografía política al estudio de la violencia y el conflicto armado en Colombia, 1990-2012. En: Cuadernos de Geografía, Bogotá, Universidad Nacional de Colombia.

SÁnchez SuÁRez, Pedro Arnulfo (CR). (2014). Poder aéreo en la seguridad y defensa nacional. En Revista Taktika $N^{\circ}$ 5. Colombia, Fuerza Aérea Colombiana.

SAntos, María Alejandra. (2014). El poder de la Fuerza Aérea Colombiana: la Operación Vuelo de Ángel y el cambio en la asimetría de poder entre las FF.MM y las FARC-EP. Bogotá, Universidad Colegio Mayor de Nuestra Señora del Rosario, Facultad de Ciencia Política y Gobierno.

VÁzQUEZ, Teófilo. (2013). El papel del conflicto armado en la construcción y diferenciación territorial de la región del Caguán, Amazonia colombiana. Medellín, Universidad San Buenaventura. 